\title{
COMPLETENESS THEOREM FOR BOOLEAN MODELS WITH STRICTLY POSITIVE MEASURE
}

\author{
Nebojša Ikodinović
}

\begin{abstract}
Rašković [3] introduce a conservative extension of classical propositional logic with some probability operators and prove corresponding completeness and decidability theorem. The aim of this paper is to prove the Robinson consistency and Craig interpolation for this logic.
\end{abstract}

\section{Introduction}

Rašković [3] introduce the logic of possibility $L P$, which is a conservative extension of classical propositional logic with same probability operators. Formulas from this logic speak about probabilities, but they remain either true or false. The language of this logic is obtained by adding probability operators to the classical propositional language. The probability operators have (in our notation) the form $P_{\geq s}$, with the intended meaning that $P_{\geq s} \varphi$ holds if the probability of $\varphi$ is greater or equal to $s$. The axiom and rules inference of the logic LP are listed in [3] for which is proved completeness with respect to "natural" models.

In this paper we investigate some probability logic $L P^{+}$which is complete with respect to Boolean models with strictly positive measure. The language of $L P^{+}$is the same as for $L P$. Axiom and rules of inference of $L P^{+}$ are those of $L P$, together with the following rule of inference:

$$
\frac{P_{\geq 1 \varphi}}{\varphi} \text {. }
$$




\section{2. $L P^{+} \operatorname{logic}$}

The symbols of $\mathrm{LP}^{+}$logic are the so-called connectives: $\wedge$ (and), ᄀ (negation), then the list of probability operators $P_{\geq s}$ for each $s \in[0,1] \cap Q$, and finally an infinite sequence of propositional letters $I$.

The set $F o r_{L P+}^{C}$ of all classical propositional formulas is defined inductively as the smallest set containing the propositional letters and closed under the usual formation rules: if $\varphi$ and $\psi$ are classical propositional formulas, then $\neg \varphi$ and $\varphi \wedge \psi$ are classical propositional formulas. The set $F_{o r}^{P}{ }_{L+}^{P}$ of all probability formulas is the smallest set such that:

- if $\varphi \in$ For $_{L P^{+}}^{C}$ and $s \in[0,1] \cap Q$, then $P_{\geq s} \varphi$ is probability formula;

- if $\Phi$ and $\Psi$ are probability formulas, then $\neg \Phi$ and $\Phi \wedge \Psi$ are also probability formulas.

Let $F_{0 P_{L+}}(I)=F_{0 r}^{C} L_{P^{+}}(I) \cup F o r_{L P^{+}}^{P}(I)$. We introduce the abbreviations $\vee, \Rightarrow, \Leftrightarrow$, in the usual way. It is convenient of use the following abbreviations in $L P^{+}$:

- $P_{<s} \varphi$ for $\neg P_{\geq s} \varphi$,

- $P_{\leq s} \varphi$ for $P_{\leq 1-s} \varphi$,

- $P_{>s} \varphi$ for $\neg P_{\leq s} \varphi$,

- $P_{=s} \varphi$ for $P_{\geq s}(\varphi) \wedge \neg P_{>s} \varphi$.

The axioms for $L P^{+}$logic are every instance of classical propositional tautology and the following ones:

(1) $P_{\geq 0} \varphi$, for all $\varphi \in$ For $_{L P^{+}}^{C}$;

(2) $P_{\leq r} \varphi \Rightarrow P_{<s} \varphi$, for all $\varphi \in F_{\text {or }}^{C}$ PP+ $^{+}$and $s, r \in[0,1] \cap Q$ such that $s>r$;

(3) $P_{<s} \varphi \Rightarrow P_{\leq s} \varphi$ for all $\varphi \in F_{o r}^{C} P_{P^{+}}$and $s \in[0,1] \cap Q$;

(4) $\left(P_{\geq r} \varphi \wedge P_{\geq s} \psi \wedge P_{\geq 1}(\neg \varphi \vee \neg \psi)\right) \Rightarrow P_{\geq \min \{1, r+s\}}(\varphi \vee \psi)$ for all $\varphi, \psi \in$ For $_{L P^{+}}^{C}, r, s \in[0,1] \cap Q$;

(5) $\left(P_{\leq r} \varphi \wedge P_{<s} \psi\right) \Rightarrow P_{<r+s}(\varphi \vee \psi)$ for all $\varphi, \psi \in F_{o r}^{C}{ }_{L+}, s, r \in[0,1] \cap Q$ such that $r+s<1$.

The rules of inference are:

(R1) From $\Phi$ and $\Phi \Rightarrow \Psi$, infer $\Psi, \Phi, \Psi \in F_{\text {or }}^{C} P_{P^{+}}$or $\Phi, \Psi \in$ For $_{L P^{+}}^{P}$.

(R2) From $\varphi$; infer $P_{\geq 1} \varphi, \varphi \in$ For $_{L P^{+}}^{C}$.

(R3) From $P_{\geq 1} \varphi$, infer $\varphi, \varphi \in$ For $_{L P^{+}}^{C}$.

(R4) From $\Phi \Rightarrow P_{\geq s-\frac{1}{k}} \varphi$, for every $k \geq \frac{1}{s}$, infer $\Phi \Rightarrow P_{\geq s} \varphi, \Phi \in$ For $_{L P+}^{P}$, $\varphi \in$ For $_{L P+}^{C}$.

A proof of formula $\Phi$ in theory $T$ of logic $L P^{+}$is every countable sequence $\Phi_{1}, \Phi_{2}, \ldots, \Phi$ of formulas such that each formula $\Phi_{i}, i<\omega$, is a axiom, or a formula from $T$, or it is derived by inference rules from preceding members of the sequence. If there exists a proof of $\Phi$ in $T$, then $\Phi$ is called a 
theorem of $T$, and in this case we use the notation $T \vdash_{L P+} \Phi$. A theory $T$ is consistent if there are any formula $\varphi \in F_{\text {or }}^{C} L_{P^{+}}$such that $T \forall_{L P+} \varphi$ and any formula $\Phi \in$ For $_{L P^{+}}^{P}$ such that $T \forall_{L P^{+}} \Phi$. A theory $T$ is a maximal consistent iff $T$ is a consistent theory and:

- for all $\varphi \in$ For $_{L P^{+}}^{C}$, if $T \vdash_{L P^{+}} \varphi$, then $\varphi \in T$ and $P_{\geq 1} \varphi \in T$;

- for all $\Phi \in$ For $_{L P^{+}}^{P}, \Phi \in T$ or $\neg \Phi \in T$.

Having in mind the deductibles relation just defined, by induction on the length of the corresponding derivation, we can prove:

Theorem 1. If $T \subseteq$ For $_{L P^{+}}$and $T \cup\{\alpha\} \vdash_{L P^{+}} \beta$, then $T \vdash_{L P^{+}} \alpha \Rightarrow \beta$, where $\alpha$ and $\beta$ are either both classical or both probability formulas.

Theorem 2. For every consistent theory $T \subseteq$ For $_{L P^{+}}$, there exists a maximal consistent theory extending $T$.

Proof. Let $T \subseteq$ For $_{L P^{+}}$be a consistent theory, $T^{c}$ the set of all classical consequences of $T$ and $\alpha_{1}, \alpha_{2}, \ldots$ an enumeration of all probability formulas from $F_{\text {or }} \mathrm{LP}^{+}$. We define a sequence of theories $T_{i}, i=0,1,2, \ldots$ such that:

1. $T_{0}=T \cup T^{c} \cup\left\{P_{\geq 1} \alpha: \alpha \in T^{c}\right\}$,

2. for every $i \geq 0$, if $T_{i} \cup\left\{\alpha_{i}\right\}$ is consistent, then $T_{i+1}=T_{i} \cup\left\{\alpha_{i}\right\}$, otherwise

3. if $\alpha_{i}$ is $\beta \Rightarrow P_{\geq s} \gamma$, and $T_{i} \cup\left\{\alpha_{i}\right\}$ is not consistent, then $T_{i+1}=$ $T_{i} \cup\left\{\beta \Rightarrow \neg P_{s-\frac{1}{n}} \gamma\right\}$, for same positive integer $n>\frac{1}{s}$, so that $T_{i+1}$ is consistent, otherwise,

4. $T_{i+1}=T_{i}$.

The set obtained by the steps 1,2 , or 4 are obviously consistent. The steps mean that $T_{i+1}$ contains a witness which guarantees that $T_{i} \cup\left\{\alpha_{i}\right\}$ is not consistent. We can show that this step produce consistent set, too. Suppose that $T_{i}$ is consistent, $\alpha_{i} \beta \Rightarrow P_{\geq s} \gamma, T_{i} \cup\left\{\alpha_{i}\right\}$ is not consistent, and for every positive integer $n, T_{i} \cup\left\{\beta \Rightarrow \neg P_{s-\frac{1}{n}} \gamma\right\}$ is not consistent. But, then

$T_{i}, \beta \Rightarrow \neg P_{s-\frac{1}{n}} \gamma \vdash_{L P+} \perp$, for every positive integer $n>\frac{1}{s}$,

$T_{i} \vdash_{L P^{+}} \neg\left(\beta \Rightarrow \neg P_{s-\frac{1}{n}} \gamma\right)$, for every positive integer $n>\frac{1}{s}$, by the deduction theorem,

$T_{i} \vdash_{L P}+\beta \Rightarrow P_{s-\frac{1}{n}} \gamma$, for every positive integer $n>\frac{1}{s}$, by the classical tautology $\neg(A \Rightarrow B) \Rightarrow(A \Rightarrow \neg B)$,

$T_{i} \vdash_{L P}+\beta \Rightarrow P_{\geq s} \gamma$, by the inference rule 4 .

This implies that $T_{i}$ is not consistent, a contradiction. Hence, every $T_{i}$ is consistent.

Let $T^{*}=\cup_{i} T_{i}$. We can prove that $T^{*}$ is a deductively closed set which does not contain all formulas, and, as a consequence, that $T^{*}$ is consistent. If $\alpha \in F_{\text {or }}^{C} L_{P^{+}}$, by the construction of $T_{0}, \alpha \neg \alpha$ cannot be simultaneously 
in $T_{0}$. For a probability formula $\alpha$ the set $T^{*}$ does not contain both $\alpha=\alpha_{i}$ and $\alpha=\alpha_{j}$, because $T_{\max (i, j)+1}$ is a consistent set. If $\alpha$ is a classical formula and $T^{*} \vdash_{L P^{+}} \alpha$, then by the construction of $T_{0}, \alpha \in T^{*}$ and $P_{\geq 1} \alpha \in T^{*}$. It can be proved by induction on the length of the inference that for every probability formula $\alpha$ if $T^{*} \vdash_{L P^{+}} \alpha$, then $\alpha \in T^{*}$. For example, let $\alpha=\alpha_{i}=$ $\left(\beta \Rightarrow P_{\geq s} \gamma\right)$ be obtained from $T^{*}$ by an application of inference rule 4 . By induction hypothesis $\beta \Rightarrow P_{\geq s-\frac{1}{n}} \gamma \in T^{*}$ for every positive integer $n>\frac{1}{s}$ and for every $n>\frac{1}{s}$ there is $T_{k_{n}}$ so that $\beta \Rightarrow P_{\geq s-\frac{1}{n}} \gamma \in T_{k_{n}}$. But, if $\alpha_{i} \notin T^{*}$, then $T_{i+1}$ and so $T^{*}$, contain $\beta \Rightarrow P_{\geq s-\frac{1}{j}} \gamma$, for an $j>\frac{1}{s}$, a contradiction. Finally, we can prove that for every probability formula $\alpha$, either $\alpha$, or $\neg \alpha$ belongs to $T^{*}$. Suppose that $\alpha$ is $\alpha_{i}, \neg \alpha$ is $\alpha_{j}, k=\max (i, j), \alpha \notin T^{*}$ and $\neg \alpha \notin T^{*}$. But, then

$$
\begin{aligned}
& T_{k}, \alpha \vdash_{L P^{+}} \perp \\
& T_{k}, \neg \alpha \vdash_{L P^{+}} \perp \\
& T_{k} \vdash_{L P^{+}} \alpha \wedge \neg \alpha .
\end{aligned} .
$$

So $T_{k}$ is not consistent, a contradiction.

Let $\mathbb{B}=(B,+, \cdot,-, 0,1)$ be arbitrary Boolean algebra. $A \mathbb{B}$-interpretation of the set $I$ of propositional letters is every map $f: I \rightarrow B$. Then, it is natural to extend the map $f: I \rightarrow B$ to map $f: F_{\text {or }_{L P^{+}}^{C}}^{C} \rightarrow B, \mathbb{B}$-interpretation of the classical propositional formulas, inductively as follows:

$$
f(\neg \varphi)=-f(\varphi), f(\varphi \vee \psi)=f(\varphi)+f(\psi), f(\varphi \wedge \psi)=f(\varphi) \cdot f(\psi) .
$$

A Boolean model for $L P^{+}$logic is every triple $(\mathbb{B}, f, \mu)$, where $\mathbb{B}$ is a Boolean algebra, $f$ is a $\mathbb{B}$-interpretation of the set of classical propositional formulas, and $\mu$ is a (finitely-additive probability) strictly positive measure on $\mathbb{B}$. For any formula $\Phi \in$ For $_{L P^{+}}$, we define the relation $(\mathbb{B}, f, \mu) \models=\Phi$, by induction on the complexity of the formulas $\Phi$, as follows:

- if $\Phi \in$ For $_{L P^{+}}^{C}$, then $(\mathbb{B}, f, \mu) \models \Phi$ iff $f(\Phi)=1$,

- if $\Phi=P_{\geq s} \varphi, \varphi \in F_{o r}^{C} P^{+}, s \in[0,1] \cap Q$, then $(\mathbb{B}, f \mu) \models \Phi$ iff $\mu(f(\varphi)) \geq$ $s$,

- if $\Phi=\Psi \wedge \Theta, \Psi, \Theta \in F_{\text {or }}^{P} P_{P^{+}}$, then $(\mathbb{B}, f, \mu) \models \Phi$ iff $(\mathbb{B}, f, \mu) \models \Psi$ and $(\mathbb{B} ; f, \mu) \vDash \Theta$,

- if $\Phi=\neg \Psi, \Psi \in F$ Fr $_{L P+}^{P}$, then $(\mathbb{B}, f, \mu) \models \Phi$ iff not $(\mathbb{B}, f, \mu) \models \Psi$

We simply write $\models \Phi$ and say that $\Phi$ is valid iff for every Boolean model $(\mathbb{B}, f, \mu),(\mathbb{B}, f, \mu) \models \Phi$.

Theorem 3. (Soundness Theorem) Any set $T$ of formulas of $L P^{+}$ logic which has a model is consistent.

Proof. As usual, to prove the soundness theorem it suffices to show that each axiom is valid and the rules of inference preserve validity. 
A classical propositional tautology is obviously valid.

Let $\Phi$ and $\Psi$ are both classical or both probability formulas such that $\Phi$ and $\Phi \Rightarrow \Psi$ are valid. If we suppose that $\not \models \Psi$, then there is a Boolean model $(\mathbb{B}, f, \mu)$ such that $(\mathbb{B}, f, \mu) \neq \Psi$ and $(\mathbb{B}, f, \mu) \models \Phi \Rightarrow \Psi$, so $(\mathbb{B}, f, \mu) \neq$ $\Phi$, which is a contradiction by validity of $\Phi$.

If $\varphi \in$ For $_{L P^{+}}^{C}$ is valid then for any Boolean model $(\mathbb{B}, f, \mu), f(\varphi)=1$, we have $(\mathbb{B}, f, \mu) \models P_{\geq 1} \varphi$.

If $P_{\geq 1} \varphi, \varphi \in F_{0} r_{L P^{+}}^{C}$, is valid then for any Boolean model, for $L P^{+}$, $(\mathbb{B}, f, \mu), \mu(f(\varphi))=1$, we have $f(\varphi)=1$, since $\mu$ is strictly positive, and so $(\mathbb{B}, f, \mu) \models \varphi$.

Finally, the rule $(R 4)$ preserve validity since the set of reals is Archimedean field.

Theorem 4. (Completeness Theorem) Every consistent theory $T \subseteq$ For $_{L P^{+}}$has a Boolean model.

Proof. Let $T$ be a consistent theory. By Theorem 1. there is a maximal consistent extension $T^{*}$ of $T$. Let $T^{c}$ be the set of all classical consequences of $T, \mathbb{B}_{T^{c}}$ the Lindenbaum algebra of $T^{c}$ and $f: F o r_{L P^{+}}^{C} \rightarrow B_{T^{c}}$ be defined by $f(\varphi)=[\varphi]_{T^{c}}$, where $[\varphi]_{T^{c}}$ is the equivalence class determined by a formula $\varphi \in F_{\text {or }}^{C}$ P $^{+}$. Let $\mu . B_{T^{c}} \rightarrow[0,1]$ be defined by:

$$
\mu\left([\varphi]_{T^{c}}\right)=\sup \left\{r \in[0,1] \cap Q: P_{\geq r} \varphi \in T^{*} \xi, \varphi \in F o r_{L P^{+}}^{C} .\right.
$$

We shall show that $\mu$ is a strictly positive measure on $\mathbb{B}_{T^{c}}$.

First, let us prove that $\mu$ is a well-defined. It is suffices to prove that for all $\varphi, \psi \in$ For $_{L P^{+}}^{C}$, if $[\varphi]_{T^{c}} \leq[\psi]_{T^{c}}$, then $\mu\left([\varphi]_{T^{c}}\right) \leq \mu\left([\psi]_{T^{c}}\right)$. Really, if $[\varphi]_{T^{c}} \leq[\psi]_{T^{c}}$, then $T^{c} \vdash(\varphi \Rightarrow \psi)$ and consequently $T \vdash_{L P^{+}} P_{\geq 1}(\varphi \Rightarrow \psi)$. Thus, if $P_{\geq s} \varphi \in T^{*}$, then $P_{\geq_{s}} \psi \in T^{*}$. So, $\mu\left([\varphi]_{T^{c}}\right) \leq \mu\left([\psi]_{T^{c}}\right)$.

It is easy to see that $\mu(1)=1$.

We show that $\mu\left([\varphi]_{T^{c}}\right)+\mu\left([\psi]_{T^{c}}\right)=\mu\left([\varphi]_{T^{c}}+[\psi]_{T^{c}}\right)$, for all $\varphi, \psi \in$ For $_{L P^{+}}^{C}$ such that $[\varphi]_{T^{c}} \cdot[\psi]_{T^{c}}=0$. Let $\mu\left([\varphi]_{T^{c}}\right)=r, \mu\left([\psi]_{T^{c}}\right)=s$. Then $r+s \leq 1$. Let us suppose that $r>0$ and $s>0$. By monotonicity, for all rational numbers $r^{\prime} \in[0, r)$ and $s^{\prime} \in[0, s)$ we have $P_{\leq \tau^{\prime}} \varphi, P_{\leq s^{\prime}} \psi \in T^{*}$. Thus, we have $P_{\geq r^{\prime}+s^{\prime}}(\varphi \vee \psi) \in T^{*}$. So, $r+s \leq \sup \left\{t \in[0,1] \cap \bar{Q}: P_{\geq t}(\varphi \vee \psi) \in\right.$ $\left.T^{*}\right\}$. If $r+s=1$, then obviously the statement holds. Let us suppose that $r+s<1$. If $r+s<t_{0}=\sup \left\{t \in[0,1] \cap Q: P_{\geq t}(\varphi \vee \psi) \in T^{*}\right\}$, then for all rational numbers $t^{\prime} \in\left(r+s, t_{0}\right), P_{\geq t}(\varphi \vee \psi) \in T^{*}$. Let us choose rational numbers $r^{\prime \prime}>r$ and $s^{\prime \prime}>s$ such that $\neg P_{\geq r^{\prime \prime}} \varphi, P_{<r^{\prime \prime}} \varphi, \neg P_{\geq s^{\prime \prime}} \psi$, $P_{<s^{\prime \prime}} \psi \in T^{*}$ and $r^{\prime \prime}+s^{\prime \prime}=t^{\prime} \leq 1$. Thus, we have $P_{\leq r^{\prime \prime}} \varphi \in T^{*}$ and we have $P_{<r^{\prime \prime}+s^{\prime \prime}}(\varphi \vee \psi), \neg P_{>r^{\prime \prime}+s^{\prime \prime}}(\varphi \vee \psi), \neg P_{>t^{\prime}}(\varphi \vee \psi) \in T^{*}$ which is a contradiction. So, $\mu\left([\varphi]_{T^{c}}\right)+\mu\left([\psi]_{T^{c}}\right)=\mu\left([\varphi]_{T^{c}}+[\psi]_{T^{c}}\right)$. Similarly, for $r=0$ and $s=0$. So, $\mu$ is a measure on $\mathbb{B}_{T^{c}}$. 
Finally, $\mu$ is a strictly positive measure since if $\mu\left([\varphi]_{T^{c}}\right)=1$, then $P_{\geq 1} \in T^{*}$ and so $\varphi \in T^{*}$, by the inference rule 3 , and $[\varphi]_{T^{c}}=1$.

It is easy to see that $\left(\mathbb{B}_{T^{c}}, f_{T^{c}}, \mu\right)$ is a Boolean model of the theory $T$.

The Boolean model of $T$ constructed in the way described above we call a canonical model.

\section{References}

[1] Zे. Mijajlović. An Introduction to model theory, Institute of Mathematics, Novi Sad, 1987.

[2] K.P.S. Rao Bhaskara and M. Rao Bhascara. Theory of charges, Academic Press, 1983.

[3] M. Rašković. Classical logic with some probability operators, Publ. Inst. Math. $53,1993,1-3$

Prirodno-matematički fakultet Radoja Domanovića 1234000 Kragujevac Yugoslavia nebojsae@eunet.yu 